\title{
Ebranler la marge dans Mol, Tituba sorcière... Noire de Salem (1986) et Memorial de Maria Moura (1992) DE RaChel de QueIROZ
}

\author{
Julie Brugier* \\ Université Paris Ouest Nanterre La Défense \\ Laboratoire Littérature et poétique comparées
}

\section{Resumé :}

Dans un effort de comparaison de Yo, Tituba la bruja negra de Salem (1986) de Maryse Condé et de Memorial de María Moura (1992) de Rachel de Queiroz, cet article veut montrer de quelle façon ces auteurs envisagent la dichotomie entre la marge et le centre. Bien qu'appartenant à des contextes historiques et culturels différents, nos deux romanciers semblent être conscients du fait que leurs créations seront reçues comme une expression de voix venues d'espaces marginaux ; de ce fait, ils créent des stratégies afin de destabiliser leurs lecteurs et de questionner le concept de marge à l'intérieur de leurs fictions, neutralisant toute tentative d'interprétation essencialiste.

Mots clefs : marginalité ; Maryse Condé ; Rachel de Queiroz ; ironie ; périphérie.

Recibido : 1 de febrero de 2016

Aprobado : 25 de abril de 2016

*Estudiante de doctorado en la Université Paris Ouest Nanterre La Défense.

Dirección electrónica: brugier.julie@u-paris10.fr 


\title{
Defying the margin in Maryse Condé's I, Tituba, Black Witch of Salem
} (1986) and Rachel de Queiroz' Memorial de Maria Moura (1992)

\begin{abstract}
By comparing Maryse Condé's I, Tituba, Black Witch of Salem (1986) and Rachel de Queiroz' Memorial de Maria Moura (1992), this article seeks to demonstrate how these authors deconstruct the dichotomy between margin and center in their novels. Although originating from different historical and cultural backgrounds, both novelists seem aware that their works will be received as an expression of voices from marginal spaces : therefore, they set up strategies to destabilize their readers and challenge the concept of margin within their fiction, negating all attempts at an essentialist interpretation.
\end{abstract}

Keywords : Marginality; Maryse Condé; Rachel de Queiroz; Irony; Periphery.

\section{Cómo citar este artículo}

APA: Brugier, J. (2016). Ebranler la marge dans Moi, Tituba sorcière... noire de Salem (1986) et Memorial de Maria Moura (1992) de Rachel de Queiroz. Poligramas, No. 42, junio. Pp.: 53-73

MLA: Brugier, Julie. "Ebranler la marge dans Moi, Tituba sorcière... noire de Salem (1986) et Memorial de Maria Moura (1992) de Rachel de Queiroz”. Poligramas 42 (2016): pp. 53-73 Print.

Chicago: Brugier, Julie. 2016. Ebranler la marge dans Moi, Tituba sorcière... noire de Salem (1986) et Memorial de Maria Moura (1992) de Rachel de Queiroz. Poligramas 42 (junio): pp. 53-73 
Le concept de marge se situe à l'intersection d'interrogations diverses dans les études littéraires : elles portent aussi bien sur des enjeux poétiques et politiques, que sur la hiérarchie des genres ou la sociologie du champ littéraire. Hétérogène, la marge semble toutefois toujours convoquer une représentation spatiale : en effet, étymologiquement, la marge, en latin margo, marginis, est le bord. Etre à la marge, ce serait se situer en bordure, à la lisière : comme il s'agit d'une position relative, la marge présupposerait donc l'existence d'un centre qui la constituerait en espace périphérique. Cette métaphore spatiale peut être replacée dans le contexte de ce que la critique anglo-saxonne a pu nommer un spatial turn dans les sciences humaines et sociales à partir des années soixante : c'est-à-dire l'émergence d'un paradigme spatial dans la théorie amorcée par le structuralisme et sa terminologie (Besse, 2010 ; Tally, 2013 ; Warf et Arias, 2009 ; West-Pavlov, 2009). Ce paradigme pourrait être résumé par cette phrase de Michel Foucault : «L'époque actuelle serait plutôt l'époque de l'espace. » (Foucault, 2001, 1571). Ce dernier a fait partie de ceux qui ont mis en évidence la dimension politique de l'espace (West-Pavlov, 38), en prenant pour objet les métaphores spatiales, qu'il appelait lui-même ses « obsessions spatiales » (Foucault, 2001, 33), afin de «saisir le processus par lequel le savoir fonctionne comme un pouvoir et en reconduit les effets » (Foucault, 2001, 34). Si nous nous permettons ce bref détour par la pensée foucaldienne, c'est précisément parce que les rapports entre l'espace, le savoir et le pouvoir nous semblent essentiels pour comprendre la façon dont la marge est problématisée dans les œuvres qui font l'objet de notre étude. Bien qu'issues de contextes historiques et culturels très différents, elles mettent toutes deux en scène des sujets marginalisés par l'histoire et semblent leur redonner une voix à travers la fiction. Le roman Moi, Tituba sorcière... Noire de Salem de Maryse Condé (1986), accompagné, dans sa première édition, du sous-titre " Histoire romanesque », reconstruit la trajectoire de Tituba Indien, une esclave originaire de la Barbade, jugée et condamnée pour sorcellerie lors du procès des sorcières de Salem en 1692. Le roman Memorial de Maria Moura de Rachel de Queiroz (1992), quant à lui, s'inspire librement de l'histoire de Maria de Oliveira, une femme qui aurait été à la tête d'une troupe de brigands 
au XVIIe siècle, dans le Nord-Est du Brésil. Toutefois, ces deux œuvres cherchent moins à réhabiliter des sujets marginalisés qu'à les mettre au service d'une déconstruction de la dichotomie marge/centre et de la logique spatiale qu'elle présuppose. Il s'agit avant tout pour ces auteures d'interroger la marge en tant qu'espace géographique et politique : les deux romancières attachent, de ce fait, une importance particulière au lieu de leur écriture et à l'horizon d'attente qu'il présuppose. Rachel de Queiroz et Condé écrivent, en effet, depuis des régions qui sont souvent tenues pour marginales (les Antilles francophones et le Nord-Est du Brésil), aussi bien du point de vue géographique, que du point de vue politique et culturel. En rendant problématique le lieu même de leur énonciation, aussi bien au sein de leurs œuvres que dans la façon dont elles en interrogent la réception, elles vont déployer des stratégies pour ébranler la marge - et cela à double titre. Elles la mettent tout d'abord en branle : c'est-à-dire qu'elles utilisent le concept de marge et de marginalité de façon stratégique dans leurs romans, en tenant compte du contexte d'écriture et de réception - chez Condé, on pourrait même parler d'une instrumentalisation de cet horizon de lecture. Ce faisant, elles ébranlent aussi le concept en faisant vaciller sa fixité, en introduisant dans leurs textes des formes d'instabilité herméneutique qui mettent à mal une approche essentialiste de la marge.

\section{Ecrire depuis la marge}

\section{Moi, Tituba : un horizon de lecture postcolonial?}

On ne saurait aborder l'œuvre de Maryse Condé sans tenir compte de sa double carrière d'universitaire et de femme de lettres. Comme l'a montré Dawn Fulton, cette position privilégiée donne à son écriture une densité critique particulière, car elle en fait le lieu d'une réflexion approfondie sur la théorie postcoloniale. Sa fiction témoigne, en effet, d'une « conscience aiguë des discussions politiques et théoriques qui entourent la production artistique des écrivains caribéens francophones » I «a keen awareness of the political and theoretical discussions surrounding the artistic production of Francophone Caribbean writers. \" (Fulton, 2008, 2. Nous traduisons.)

Dès lors, et en prenant acte du fait que l'œuvre Condé a souvent été analysée dans une perspective postcoloniale, il est nécessaire de revenir 
sur la façon dont la marge et la marginalité sont conceptualisées dans la théorie postcoloniale. Dans le contexte postcolonial, ces concepts ont en premier lieu un sens géographique dont les implications sont politiques : elles renvoient à la condition périphérique des anciennes colonies par rapport aux métropoles - et par extension, à celle des colonisés vis-à-vis des colonisateurs. Cette condition périphérique, marginale, est avant tout une construction : elle dérive d'une idéologie qui cherche à donner une position centrale aux colonisateurs. Ainsi, comme le montrent Bill Ashcroft, Gareth Griffiths et Helen Tiffin : « La marginalité est une condition induite par la relation à un centre privilégié, une «Altérisation » imposée par l'autorité impériale. » $(2012,127)$

La littérature postcoloniale, émergeant de ces périphéries créées par la colonisation, peut se définir, selon ces auteurs, comme toute la littérature affectée par le processus impérialiste depuis le moment de la colonisation jusqu'à la période contemporaine. Ils soulignent que ce qui se dégage de commun aux diverses littératures regroupées sous cette étiquette est une réflexion commune sur ce partage entre marge et centre. Les trois caractéristiques majeures de toute la littérature postcoloniale seraient donc ainsi :

[L]e bâillonnement et la marginalisation de la voix postcoloniale par le centre impérial ; l'abrogation de ce centre impérial à l'intérieur du texte ; enfin, l'appropriation de la langue et de la culture même de ce centre [...] Dans tous les cas, cependant, les notions de pouvoir inhérentes au modèle centre/marge sont appropriées et donc démontées (Aschroft, Griffiths et Tiffin, 2012, 104)

Le texte postcolonial serait donc le lieu privilégié d'une « contreécriture ", c'est-à-dire d'un renversement des dynamiques de pouvoir qui ont présidé à la constitution de ce partage entre marge et centre. Ce renversement se ferait, entre autres, par un dialogue critique avec l'histoire, en montrant comment des sujets au statut de marginaux ont été réduits au silence et à l'oubli. La tâche de l'écrivain et du critique contemporain serait alors, selon Homi K. Bhabha, de « réaliser pleinement les passés non dits, non représentés, qui hantent le présent historique, et d'en assumer la responsabilité. »(Bhabha, 2007, 46) 
La possibilité de redonner voix aux sujets marginalisés par l'histoire devient donc l'horizon politique de cette écriture.

Le roman Moi, Tituba, sorcière... Noire de Salem, publié en 1986 par Maryse Condé, semble s'inscrire parfaitement dans cette dynamique de renversement du dispositif centre/marge hérité des rapports de domination coloniale. En effet, ce roman, dont l'histoire se situe en Barbade puis à Boston et à Salem, est l'autobiographie fictive d'un personnage historique ayant réellement existé : Tituba Indien, l'esclave barbadienne d'un pasteur puritain, jugée lors du procès des sorcières de Salem, en 1692. Elle fut la première femme dans l'histoire du procès à confesser avoir pratiqué de la sorcellerie ; contrairement à d'autres accusées, elle ne fut pas condamnée à mort et fut peut-être vendue à un marchand d'esclaves. On perd historiquement la trace de Tituba après sa sortie de prison, alors que ce n'est pas le cas pour les autres « sorcières » de Salem. Le roman de Condé aurait émergé de la prise de conscience du biais idéologique de l'historiographie. Dans une « note historique » qui suit son roman, elle attribue en effet l'oubli de Tituba au racisme et fait de la fiction le lieu où peut se réécrire l'histoire :

Vers 1693, Tituba, notre héroïne, fut vendue pour le prix de sa « pension » en prison, de ses chaînes et de ses fers. A qui ? Le racisme, conscient ou inconscient, des historiens est tel qu'aucun ne s'en soucie. [...] Une vague tradition assure qu'elle fut vendue à un marchand d'esclaves qui la ramena à la Barbade. Je lui ai offert, quant à moi, une fin de mon choix. (Condé, 2012, 277-278)

Ce projet est explicite dans le titre de son ouvrage : Moi, Tituba, sorcière... Noire de Salem. On a là la mise-en-scène emphatique d'une énonciation à la première personne, avec le pronom personnel détaché au début du titre, suivi immédiatement du prénom du personnage principal. Condé semble souligner ainsi ce désir de redonner voix à un sujet historiquement marginalisé, en lui donnant l'autorité narrative et en opérant à travers la fiction un recentrement (Fulton, 2008, 39-40). En outre, cette prise de parole du marginal a réellement lieu dans le roman puisque Condé y retranscrit un document historique : l'interrogatoire de Tituba Indien, qu'elle a traduit de l'anglais. La transcription et la 
traduction sont fidèles au document original, presque entièrement rapporté dans le livre'. L'auteure incite ainsi son lecteur à entendre la voix de la Tituba historique, oubliée ou déformée par l'historiographie.

Toutefois, le titre n'est pas celui que Condé souhaitait donner originellement à son œuvre. Dans la postface à l'édition américaine, l'auteure affirme qu'elle voulait l'intituler simplement Moi, Tituba mais que « les éditeurs trouvaient cela un peu laconique pour un titre et ils ont ajouté sorcière... Noire de Salem »/ «but the publishers said that was a bit laconic as a title and added Black Witch of Salem. » (Condé, 1992, citée dans Manzor-Coats, 1993, 738. Nous traduisons.) L'ajout des épithètes « sorcière... Noire de Salem » surdétermine les caractéristiques marginales de Tituba ou, plus précisément, cherche à exagérer l'exotisme de l'œuvre pour exploiter l'ethnicité de Condé (Marouan, 2013, 138). Il témoignerait d'une stratégie éditoriale consistant à programmer la réception du livre comme un roman postcolonial réinventant l'histoire d'un sujet marginalisé ${ }^{2}$. On pourrait objecter à cela le fait que le titre de la dernière édition ait fait disparaitre « Noire de Salem » de la couverture : l'expression n'apparaît plus que sur la page de garde, avec l'ajout des points de suspension absents de la couverture de la première édition. Cependant, le tableau de la peintre Marie-Guillemine Benoist, "Portrait d'une négresse » (1800), en couverture de l'édition Folio de 1988, montre une femme noire, drapée dans un tissu blanc, le regard dirigé vers le spectateur : il viendrait ainsi se substituer à la deuxième partie du titre, indiquant l'origine de Tituba. Marie-Guillemine Benoist a présenté ce tableau lors du salon de 1800 en prenant pour sujet une domestique noire, probablement une ancienne esclave originaire de Guadeloupe. Il est encore rare, au moment où cette peinture est réalisée, de représenter des Noirs autrement qu'à l'arrière-

${ }^{1}$ Toutes les archives du procès des sorcières de Salem ont été mises en ligne par l'Université de Virginie, aux Etats-Unis : http ://salem.lib.virginia.edu/ home.html. Pour consulter l'interrogatoire de Tituba Indien : http ://salem.lib. virginia.edu/texts/tei/swp?term=Tituba\&div $\mathrm{id}=\mathrm{n} 125.4 \&$ chapter $\mathrm{id}=\mathrm{n} 125$.

${ }^{2}$ Lilian Manzor-Coats a proposé une analyse détaillée de la façon dont cette stratégie est adaptée dans la traduction étasunienne ; elle montre en particulier comment la préface d'Angela Davis a pu servir une stratégie de légitimation féministe de l'œuvre pour le marché étasunien (Manzor-Coats, 1993, 739). 
plan des tableaux, en train de servir des maîtres blancs : en ce sens, ce portrait que fait Marie-Guillemine Benoist d'une « négresse » dans l'attitude d'une femme noble est atypique (Smalls, 2004) et semble chercher à lui redonner une dignité que lui dénie la société. On pourrait y voir alors un parallèle avec la démarche que Condé revendique dans sa « Note historique»: redonner une place centrale à un personnage historiquement ou socialement marginal. Cette femme reste anonyme et cet anonymat, ainsi que l'origine guadeloupéenne de la «négresse », invitent à lire dans ce portrait une double allusion : à Tituba mais aussi à Maryse Condé (Marouan, 2013, 138).

Cette stratégie éditoriale nous invitant à lire le roman comme une réécriture de l'histoire se retrouve également dans les textes sur la quatrième de couverture des différentes éditions. En effet, dans la première édition française, qui date de 1986, la quatrième de couverture résume le livre en disant que "Maryse Condé a choisi de nous parler de Tituba que l'Histoire a oubliée pour ne retenir que les sorcières blanches, celles qui furent pendues et inspirèrent Les Sorcières de Salem, d'Arthur Miller» (Condé, 1986). Dans l'édition Folio de 1988, le texte surenchérit sur la réhabilitation de Tituba : «Tituba est arrêtée, oubliée dans sa prison jusqu'à l'amnistie générale [...]. Là s'arrête l'histoire. Maryse Condé la réhabilite, l'arrache à cet oubli auquel elle avait été condamnée » (Condé, 2012). Rien d'étonnant, dès lors, à ce que l'histoire de Tituba puisse être lue et reçue, pour reprendre l'expression de Dawn Fulton, comme :

the quintessential portrait of a marginalized subject : she is a slave and a woman, victimized by a colonial economy and seemingly overlooked by historical record. (Fulton, 2008, 39)

la quintessence du portrait du sujet marginalisé : elle est esclave et femme, victime de l'économie coloniale et en apparence oubliée par les archives historiques. (Nous traduisons.)

Rachel de Queiroz et l'invention du Nordeste marginal

Le roman de Rachel de Queiroz, quant à lui, n'appartient pas à un contexte postcolonial mais à la littérature du Nord-Est brésilien, le Nordeste ; l'auteure était elle-même originaire du sertão ${ }^{3}$ du Ceará, un

${ }^{3}$ Région semi-aride située dans l'intérieur des terres de la région Nord-Est du Brésil. 
des états de cette région. Or, le Nordeste s'est construit, à maints égards, comme un espace de marginalité et d'altérité dans l'imaginaire brésilien — valorisant, par contraste, le Sud et Sud-Est du pays comme centres moteurs du progrès et de la modernité (Rago, 1999, 14). Dans un essai intitulé A invenção do Nordeste e outras artes, l'historien Durval Muniz de Albuquerque Jr. étudie la façon dont cette région s'est produite et construite historiquement et culturellement et montre les relations de pouvoir qui sous-tendent ce découpage spatial — projet qui rappelle, évidemment, celui d'Edward Saïd dans L'Orientalisme (1978). En s'appuyant sur Foucault, Albuquerque entreprend d'historiciser et de déconstruire les discours qui ont rendu visible et énonçable le Nordeste comme région. La région s'élabore à travers des discours qui contribuent à en faire un espace de marginalité :

Este saber sobre a região, saber estereotipado, [...] reserva a este espaço o lugar do gueto nas relações sociais em nível nacional, região que é preservada com elaboração imagético-discursiva como o lugar da periferia, da margem, nas relações econômicas e políticas no país, que transforma seus habitantes em marginais da cultura nacional. (Muniz de Albuquerque Jr., 1999, 27)

Ce savoir sur la région, stéréotypé, [...] la situe dans l'espace du ghetto dans les relations sociales à échelle nationale, région qui se maintient à travers l'élaboration d'un ensemble de discours et d'images, comme l'espace de la périphérie, de la marge, dans les relations économiques et politiques au sein du pays, qui transforme ses habitants en marginaux vis-à-vis de la culture nationale. (Nous traduisons.)

Il est intéressant de noter que cet essai s'ouvre et se clôt sur l'analyse d'une expression utilisée par Rachel de Queiroz. Dans un article de 1988, celle-ci dénonçait le « regard tordu » des médias sur le Nordeste, en les accusant de véhiculer une image misérable de la région. Or, pour Albuquerque, l'auteure aurait elle-même contribué à construire ce « regard tordu » car il émerge non seulement de l'extérieur de la région, mais aussi des discours produits et reproduits par le peuple nordestino lui-même - et en cela l'analyse de Albuquerque diffère de celle de Saïd qui insiste sur l'extériorité des discours qui ont produit l'Orientalisme. S'il choisit Queiroz pour illustrer un double discours régionaliste, lequel dénonce la représentation du Nordeste tout en contribuant à sa 
marginalité, c'est précisément parce qu'elle est une auteure dont la réception a été fortement conditionnée par l'étiquette " régionaliste » qui lui a été accolée dès son premier roman, publié en 1930.

L'Année de la grande sécheresse (O Quinze, 1930), traite en effet d'un épisode historique de sécheresse dans le Ceará — or, ce sujet, pour Albuquerque, est celui qui a le plus contribué à donner une visibilité à la région tout en lui fabriquant une altérité, une marginalité à l'échelle nationale. A l'étiquette régionaliste, s'ajoute celle d'une écriture qui aurait été « féminine », à défaut d'être féministe, ce dont Queiroz s'est toujours défendue avec véhémence ${ }^{4}$. Si Queiroz rejette, comme nombre de ses contemporains, le titre d'auteur régionaliste, jugé réducteur, elle n'en revendique pas moins ouvertement une nordestinité ainsi qu'une féminité de son approche. Elle se positionne dès lors dans une double marginalité par rapport au champ littéraire brésilien. Dans des propos rapportés par Heloisa Buarque de Hollanda, elle expose ainsi l'importance pour elle de ce lieu d'écriture décrit comme un lieu de la différence, de l'écart :

O meu approach é o da mulher totalmente integrada na vida nordestina. Eu assumo isso em todos os meus personagens. Eu não sou uma pessoa deslocada, sou aquela que não sai de lá mesmo quando sai. Essa diferença eu me reservo e cobro dos outros quando me confundem com a tropa geral dos literatos, eu me isolo disso. Realmente, meu ângulo é feminino, é pessoal. (Queiroz citée par Buarque de Hollanda, 1997, 114)

Mon approche est celle de la femme totalement intégrée dans la vie nordestina. J'assume cela dans tous mes personnages. Je ne suis pas une personne déplacée, je suis celle qui ne sort pas de là-bas même quand elle en sort. Cette différence, je me la réserve et je l'exige des autres quand ils me confondent avec la troupe des lettrés, je me mets à l'écart de tout cela. Vraiment, mon approche est féminine, elle est personnelle. (Nous traduisons.)

\footnotetext{
${ }^{4}$ «Eu sempre tive horror das feministas ; elas até me chamavam de machista. Eu acho o feminismo um movimento mal orientado. Por isso sempre tomei as providências para não servir de estandarte para ele. » (Queiroz, 1997, 26) / « J'ai toujours eu horreur des féministes ; elles disaient même que j'étais machiste. Je considère le féminisme un mouvement très mal orienté. C'est pourquoi j'ai toujours pris des mesures pour ne pas lui servir d'étendard. » (Nous traduisons.)
} 
Cet écart, comme nous pouvons le noter, se dit moins par rapport à sa région qu'à une certaine élite intellectuelle avec laquelle elle refuse d'être confondue. La nordestinité et la féminité deviennent alors des lieux stratégiques pour exprimer la singularité de sa plume. Cette auteure, qui revendique ce décalage, finit sa carrière de romancière à quatre-vingts deux ans avec Memorial de Maria Moura. Ce roman pourrait être $\mathrm{lu}$, à maints égards, comme une apothéose $\mathrm{du}$ discours d'invention d'un Nordeste marginal, clôturant un cycle initié soixantedeux ans plus tôt avec L'année de la grande sécheresse. Queiroz retrace l'histoire d'une jeune femme, Maria Moura, laquelle, dans l'intérieur des terres du Ceará au XIXe siècle, devient un redoutable bandit, à la tête d'une troupe de cangaceiros, des hors-la-loi du Nordeste du Brésil.

L'œuvre engage, en effet, un dialogue avec l'histoire régionale. Le titre brésilien, Memorial de Maria Moura inclut une référence au genre des mémoires et nous invite donc à la lire comme un mélange de récit intime et de récit historique dans le cadre de la fiction. Cette proximité entre fiction et histoire évoque le projet de Condé ; Queiroz explique d'ailleurs dans un entretien qu'elle s'est inspirée d'un personnage historique réel. Il s'agit de Maria de Oliveira, qui, lors d'une sécheresse en 1602, est « devenue célèbre parce que, avec ses enfants et quelques gars, elle s'est mise à piller des fermes. » / « tornou-se conhecida, porque juntamente com os filhos e uns cabras, saiu assaltando fazendas. » (Queiroz, 1997, 34. Nous traduisons.) Queiroz décrit cette femme comme une "Lampiona de l'époque » / " a "Lampiona" da época » (Queiroz, 1997, 34. Nous traduisons) : c'est-à-dire comme le pendant féminin du célèbre cangaceiro Lampião, dont la légende a été largement diffusée dans le Nordeste. Le cangaceiro est une figure topique de la littérature du Nordeste, un personnage de marginal ambigu qui incarne à la fois une révolte contre les puissants mais aussi la lutte contre la modernisation de la région. En choisissant de traiter du cangaço, sujet qu'elle avait déjà abordé en 1953 dans une pièce intitulée Lampião, et en situant son récit dans un sertão ${ }^{5}$ archaïque, traversé par des querelles de terres et d'honneur et peuplé de personnages qui renvoient à des tropes de la littérature régionale (des saltimbanques, un beato ${ }^{6}$, des bandits,

${ }^{5}$ Cf. supra.

${ }^{6}$ Littéralement, un béat ou un bigot. Dans le Nordeste, prêcheur fanatique qui sillonne l'inté- 
des familles puissantes), Queiroz s'inscrirait dans la lignée d'évocations nostalgiques et traditionnalistes du Nordeste. Elle contribuerait ainsi à renforcer son altérité dans le discours national. Toutefois, chez Queiroz comme chez Condé, ce positionnement n'est jamais caricatural et les deux auteures font de leur fiction le lieu où vont se déployer des stratégies pour ébranler une approche essentialiste de la marge.

\section{Ecrire contre la marge}

\section{La marge comme piège herméneutique dans Moi, Tituba}

Maryse Condé est très consciente de sa position dans le champ de la littérature mondiale et joue avec l'horizon d'attente qui l'accompagne. Dans une nouvelle qui s'intitule "Trois femmes à Manhattan » (Condé, 1982), elle met en scène un personnage, Élinor qui, frustrée des critiques que reçoit son livre, s'exclame : "Ils veulent que je parle une fois de plus d'esclavage et traite et racisme, que je nous pare des vertus des victimes, que j'insuffle l'espoir... » (Condé, 1982, 313) Il s'agit ici d'une critique du public afro-américain : ceux qui reprochent à Elinor de ne pas aborder les sujets qu'on attendrait d'une auteure afro-américaine. Mais Condé, par l'intermédiaire de son personnage, vise aussi, plus largement, tout l'horizon d'attente que convoque son écriture. Dans une série d'entretiens accordés à Françoise Pfaff, elle commente la phrase de son personnage et explique le double piège dans lequel tombent les écrivains de la diaspora noire, réduits par les éditeurs et le public à certains genres ou sujets comme l'esclavage et le racisme :

C'est un piège dans lequel les écrivains de la diaspora noire tombent souvent parce que nous avons le malheur d'être publiés par l'Autre. L'Autre nous enferme dans une image, nous saisit dans un regard, et nous n'avons pas toujours la possibilité de dire « non » et de nous présenter autrement. [...] A travers cette phrase que tu cites, Élinor se plaint surtout des gens de sa propre communauté qui, eux aussi, exigent de l'écrivain qu'il se conforme à certains canons. L'écrivain noir se trouve dans une situation très difficile : d'une part, il a son peuple, les siens, ceux qui le lisent localement qui demandent et attendent une certaine chose, et puis d'autre part il y a la presse qui fait le succès

rieur des terres en lisant des extraits de la Bible et en faisant des prophéties. 
littéraire, qui chosifie, exotifie et demande autre chose. Il faut donc se retrouver entre ces deux types d'exigences. (Pfaff, 1993, 149)

Or, quatre ans après la publication de cette nouvelle, quand Mme Gallimard demande à Condé de « faire un livre sur une héroïne de [s] a région.» (Pfaff, 1993, 88), elle refuse le projet avant de lui soumettre quelque temps plus tard Moi, Tituba - un livre qui semble correspondre très exactement au «piège» mentionné dans l'entretien. Le piège se retourne ici contre cet «Autre» qui la publie mais aussi contre le lecteur. Condé sait, en effet, que ses livres peuvent être lus comme l'expression d'une voix marginale (ou périphérique) sur la scène littéraire mondiale — et elle sait sans doute aussi que cela fait partie des stratégies éditoriales pour vendre ses romans. Comme le souligne Dawn Fulton, la littérature écrite par des femmes qui se situent hors de la culture dominante est souvent reçue par le lectorat dominant avec des attentes stéréotypées, qui font que « le texte écrit par une voix subalterne porte la catégorie marginale comme seul moyen d'interprétation disponible.» / « the text written by a subaltern voice carries its marginal category as the only available means of interpretation. » (Fulton, 2008, 49. Nous traduisons.)

Condé instrumentalise cet horizon d'attente et conduit à dessein le lecteur dans le piège d'une lecture au premier degré - et ce de façon très efficace, puisque dans un premier temps son livre est pris au sérieux. De nombreux critiques ont ainsi voulu faire du roman un exemple parfait d'un discours contre-hégémonique, qui redonnerait, à travers l'écriture, une place centrale aux marges de l'histoire (Moss, 1999, 2). Or, l'auteure affirme, dans des entretiens, qu'elle ne comprend pas les raisons de cette mauvaise interprétation et s'en étonne : «Je ne vois d'ailleurs pas comment des gens ont pu lire Moi, Tituba sorcière... Noire de Salem avec sérieux et au premier degré, et faire de Tituba ce qu'elle n'est pas. » (Pfaff, 1993, 90) Rien ne nous oblige, toutefois, à croire son étonnement. En effet, en induisant ses lecteurs en erreur puis en explicitant a posteriori son intention parodique, Condé les oblige à prendre conscience de leurs propres attentes. Fascinés par l'histoire de Tituba, curieux de lire ce qu'aurait pu raconter ce personnage marginalisé, nous nous retrouvons finalement dans la même position 
que ses éditeurs, toujours prêts à accepter ses manuscrits, selon elle, parce que «les gens étaient curieux de voir ce qu'une femme noire avait à dire, ce qu'elle avait dans la tête. » (Pfaff, 1993, 41)

Condé cite comme exemple de son intention parodique la rencontre qu'elle imagine entre Tituba Indien et Hester Prynne, l'héroïne du roman de Nathaniel Hawtorne, La lettre écarlate, publié en 1850. L'invraisemblance de leur rencontre, mélangeant un personnage d'une fiction extérieure et un personnage historique, se double d'anachronismes. Ainsi, dans un dialogue que les personnages ont en prison, Hester déplore le fait qu'elle ne fera jamais de Tituba une «féministe» - mot que Tituba ne comprend pas et qu'Hester ne lui explique pas, la sommant de se taire et promettant de l'expliciter plus tard. Ce passage a souvent été interprété comme une critique de la façon dont le féminisme blanc anglo-saxon a établi des relations de domination avec les femmes de couleur, à partir d'un présupposé d'universalité qui a réduit au silence les différences au sein des mouvements féministes (Duffey, 1996; Moss, 1999 ; Marouan, 2013). C'est une des interprétations possibles de ce dialogue - mais, pour l'appréhender différemment, il faut le replacer dans la diégèse. Il se situe avant la retranscription de l'interrogatoire réel de la Tituba historique et suit un extrait où Hester lui explique ce qu'elle doit répondre aux juges puritains. Dans un passage qui précède ce dialogue, Hester dit à Tituba d'exagérer tous les attributs que les puritains confèrent aux sorcières :

— Fais-leur peur, Tituba ! Donne-leur-en pour leur argent ! Décrisle [Satan] sous la forme d'un bouc avec un nez en forme de bec d'aigle [...]. Qu'ils tremblent, qu'ils frémissent, qu'ils se pâment ! [...] J'éclatais de rire :

— Voyons, Hester, tout cela est ridicule !

— Mais puisqu'ils y croient! Que t'importe, décris ! (Condé, 2012, 158).

Cet extrait peut se lire de façon méta-textuelle comme une adresse au lecteur : la critique y a vu une façon de montrer que le livre et l'interrogatoire historique de Tituba sont tous deux des performances destinées à un regard et un lectorat blancs. Le lecteur va trembler, frémir, se pâmer au spectacle de la voix de Tituba, ressuscitée d'entre 
les morts, extirpée de l'oubli par Condé : or, la Tituba créée par Condé parodie une " marge » tout comme la véritable Tituba a peut-être parodié la " sorcière ", en parlant de balais volants, de sabbats et de torture d'enfants afin d'échapper à l'exécution (Fulton, 2008, 51). La rencontre entre Hester et Tituba est tout aussi « ridicule », parodique, mais « puisqu'ils y croient », certains lecteurs la prennent suffisamment au sérieux pour y lire une confrontation entre une voix subalterne et une voix hégémonique.

Toutefois, une autre interprétation semble s'imposer. En préparant notre lecture de l'interrogatoire de Tituba par cet échange, et en l'entrecoupant à dessein d'aposiopèses et de commentaires comme «Cela dura des heures. J'avoue que je n'étais pas une bonne actrice », Condé signale sa propre instrumentalisation de la voix de Tituba. Ainsi, par exemple, elle fait prédire au personnage de Tituba son propre oubli historiographique :

Aucune, aucune biographie attentionnée et inspirée recréant ma vie et ses tourments !

Et cette future injustice me révoltait ! Plus cruelle que la mort ! (Condé, 1992, 173)

L'exclamation de Tituba est à prendre sur le ton de l'ironie : si le lecteur s'attend à trouver dans son roman une « biographie attentionnée et inspirée », recréant les tourments de la Tituba historique, il risque fort de se tromper. En outre, l'oubli historiographique que prédit le personnage et auquel la note historique prétend l'arracher est tout relatif : en effet, depuis le procès, de nombreux historiens et écrivains se sont intéressés à Tituba et son rôle dans le procès des sorcières de Salem (Jalaizal, 2009). Cet extrait, ainsi que la conversation entre Hester et Tituba, nous invitent à revenir sur la première épigraphe du roman où Condé déclare :

Tituba et moi, avons vécu en étroite intimité pendant un an. C'est au cours de nos interminables conversations qu'elle m'a dit ces choses qu'elle n'avait confiées à personne. (Condé, 2012) 
Cette épigraphe est elle aussi ironique : Condé invente une fiction d'inspiration, alors même qu'elle affirme par ailleurs que tout ce qui touche au surnaturel dans le roman relève de la dérision (Pfaff, 1993, 90). Ces « interminables conversations » qu'elle évoque doivent être mises en parallèle avec le dialogue entre Hester et Tituba. Celui-ci permet, en effet, de comprendre que l'interrogatoire de Tituba est une performance. De même, Condé signale peut-être ici que l'écriture du roman instrumentalise aussi une voix historiquement subalterne, pour ébranler les a priori herméneutiques du lecteur. Cette voix devient alors inaudible, remplacée par la parodie.

\section{Une marge qui s'abolit dans le centre : Memorial de Maria Moura}

Si Rachel de Queiroz ne déploie pas la même ironie que Maryse Condé, elle ne problématise pas moins son propre lieu d'écriture. L'histoire de Maria Moura n'est ni la geste héroïque d'une cangaceira justicière, personnifiant le Nordeste de la révolte sociale, ni celle d'une cangaceira barbare, faisant du Nordeste une terre de sang et de violence - deux aspects de la représentation du cangaço soulignés par Durval Muniz de Albuquerque Jr. Ce qui permet de comprendre la portée que donne l'auteure à son récit est plutôt sa dédicace étonnante : " A S.M. Elisabeth I, Rainha da Inglaterra (1533-1603), pela inspiração » (Queiroz, 1992) / « A S. M. Elisabeth I, Reine d'Angleterre (1533-1603) pour l'inspiration » (Queiroz, 2009). Dans un entretien, Queiroz affirme qu'elle a toujours admiré cette reine anglaise et que, trouvant qu'elle ressemblait à Maria de Oliveira, elle s'est mise à mélanger les deux personnages historiques (Queiroz, 1997, 34) - et la critique a relevé, effectivement, des similitudes entre l'histoire de la reine vierge et le récit de Maria Moura, notamment autour de la question du mariage et de la quête de légitimité (Schpun, 2002, 177-179). Mais le parallèle pourrait être approfondi : il faut aussi noter que le règne d'Elisabeth $\mathrm{I}^{\mathrm{e}}$ correspond au moment où débute l'expansion impériale anglaise, avec la découverte de nouvelles terres et l'affirmation de l'Angleterre comme puissance européenne. Nous pouvons émettre l'hypothèse que Rachel de Queiroz a choisi cette monarque habile 
et autoritaire du $\mathrm{XVI}^{\mathrm{e}}$ siècle anglais comme modèle d'écriture d'un personnage de hors-la-loi dans le Nordeste du XIX ${ }^{\mathrm{e}}$ siècle précisément pour ce qu'elle permet de comprendre sur les rapports entre marge et centre. En effet, à travers cette allusion décalée, Queiroz semble montrer la façon dont la marge est inévitablement attirée par le centre, dont elle est vouée à devenir un nouveau centre et par là-même à s'abolir.

Or l'histoire de Maria Moura peut être lue comme un récit sur les origines Brésil, s'éloignant donc de l'histoire régionale (Courteau, 2001) : partie à la conquête d'une ancienne concession héritée d'une lointaine ancêtre portugaise d'origine noble, elle s'assimile aux conquérants qui explorent le territoire, bandeirante décalée qui croise sur son chemin une communauté de marrons, des indiens et un garçon appelé Païen. Dans une terre qui s'appelle Serra dos Padres, et qui évoque aussi bien les jésuites qui ont participé à la colonisation du Brésil que le Trésor de Sierra Madre de John Huston (1948), elle fonde, selon les instructions de son père, une maison qu'elle baptise Casa-Forte (et dans laquelle on peut entendre à la fois le château fort et la casa grande, la maison des maîtres). Cette maison serait un centre de pouvoir qui rivaliserait avec la Casa da Torre d'Ávila, château-fort brésilien bien réel, construit au XVIe siècle, et qui pendant deux-cent cinquante ans fut le principal siège de pouvoir dans le Nordeste. Loin de menacer ce type de pouvoir, la Casa Forte de Maria Moura viendrait s'y substituer imaginairement, au moment de son déclin :

Maior do que a Casa Forte de Maria Moura, só a Casa da Torre - e essa mesma o povo diz que já se acabou, na Bahia. (Queiroz, 1992, 421)

Plus puissante que la Casa Forte de Maria Moura, il n'y a guère que la Casa da Torre à Bahia - et celle-là, la rumeur dit qu'elle n'en a plus pour longtemps. (Queiroz, 2009, 451).

Comme elle évoque cet ancien centre d'un pouvoir patriarcal et oligarchique, rappelant le Brésil colonial, la Casa Forte pourrait rejoindre un imaginaire du Nordeste comme espace de la saudade, c'est-à-dire de la nostalgie d'un passé aboli et d'une sociabilité antimoderne. Queiroz ferait alors de la Casa Forte un rempart contre la modernisation du pays, inventant un nouveau centre de pouvoir fictif : la marge s'annulerait 
dans ce centre, lui-même déjà anachronique, marginal, dans le contexte du Brésil impérial. L'espace serait peut-être alors chez Queiroz, « la dimension conservatrice de la vie » / " a dimensão conservadora da vida », (Muniz de Albuquerque Jr., 1999, 83. Nous traduisons.). Un élément de l'épitexte du roman permettrait toutefois de nuancer cette affirmation, dans la mesure où il relève d'un imaginaire spatial qui engage encore une fois une réflexion sur la dichotomie marge/centre. Il s'agit d'un croquis fait par l'auteure, qu'elle insère au milieu de son œuvre : il représente l'agencement des pièces de la Casa Forte. Dans ses manuscrits, Queiroz fait souvent appel à des dessins, croquis et photographies, mais ce n'est que dans Memorial de Maria Moura qu'elle publie une de ces images. Andrea Cristina de Martins Pereira (2008), auteure de la seule étude génétique qui ait été faite sur cette œuvre, insiste sur l'importance de ce dessin que l'auteure ébauche à plusieurs reprises et qu'elle fait même refaire par un ami architecte. Il s'agit avant tout pour Queiroz de souligner l'importance d'une pièce en particulier dans la maison : le cubico. Le cubico, est une pièce cachée, dissimulée par un jeu de parois décalées et attenantes à la chambre de Maria Moura. La trappe qui y donne accès ne s'ouvre que par une serrure à secret et est occultée par un coffre. Le personnage fait construire cet espace dissimulé d'après les instructions de son père, qui évoquait une pièce semblable dans la maison de ses parents. A sa mort, il lui lègue le dessin de la pièce, et le croquis de Queiroz est présenté dans le roman comme une version modifiée de celui légué par le père. La pièce, dont le nom est forgé par déformation à partir de cubículo (mot qui désigne une petite chambre ou la cellule d'un religieux), dispose d'un fond factice qui permet de cacher l'or des pillages ; mais elle sert surtout à donner l'asile à ceux qui le demanderaient ou à garder emprisonné un ennemi. Cette pièce, au centre de la Casa Forte, est ce qui fonde le pouvoir de Maria Moura, et elle parle des « vertus du cubicule » (Queiroz, 2002, 338) / « resultado do cubico » (Queiroz, 1992, 364) et estime que grâce à cette pièce " il ne faudrait pas longtemps pour qu'on me traite de sorcière... » (Queiroz, 2002, 338) / « com pouco eu ia ganhar era fama de feiticeira » (Queiroz, 1992, 364). Son pouvoir est donc fondé sur cette pièce creuse, un espace vide qui lui sert d'instrument de pouvoir 
justement parce qu'il est occulté, caché, en marge de l'architecture de la Casa Forte - et, en même temps, très visible aux yeux du lecteur ayant accès au croquis. Peut-être pourrait-on voir dans la fascination de la Moura mais aussi de Queiroz pour cet espace, une mise à mal de ce nouveau centre. Une fois construit, il menace encore de se dérober et de disparaître, comme le signalent ses parois qui se décalent et le déplacent dans l'espace fictif construit par le texte.

L'attention toute particulière que nos auteures portent à la marge comme lieu même de leur écriture, ou, plutôt, comme lieu auquel leur écriture est assignée, invite le lecteur à dépasser une lecture qui essentialiserait la marge. Leurs romans mettent bien en évidence la façon dont cette dichotomie spatiale marge/centre, aux enjeux politiques et idéologiques, crée des positions parfois caricaturales et dont elles cherchent à s'éloigner. En écrivant contre la marge, et nous serions même tentés de dire tout contre la marge, au plus près de celleci, elles font de leurs fictions un espace où le concept de marge peut être déconstruit. Dans le roman de Maryse Condé, sans doute plus que dans celui de Queiroz, s'élabore alors une propédeutique de lecture. En effet, son œuvre n'invalide jamais complètement une interprétation au premier degré : et peut-être est-il nécessaire de tomber dans le piège d'une lecture qui essentialise la marge pour pouvoir comprendre comment celle-ci se construit et comment elle est instrumentalisée. Nous pourrions alors relire l'exclamation du personnage d'Hester, s'adressant à Tituba, comme une exclamation de Condé elle-même, raillant ses lecteurs, dont elle sait bien qu'ils seront pressés de croire à la réhabilitation qu'elle prétend mener : «-Mais puisqu'ils y croient ! Que t’importe, [d]écris !» (Condé, 2012, 158). 


\section{Bibliographie}

Ashcroft, B., Griffiths, G. et Tiffin, H. (2012) [1éd. 1989]. L'Empire vous répond : théorie et pratique des littératures post-coloniales (traduit par J.Y. Serra et M. Mathieu-Job). Pessac, France : Presses Universitaires de Bordeaux.

Besse, J.-M. (2010). Approches spatiales dans l'histoire des sciences et des arts. L'Espace Géographique, 2010/3 (39), 211-224.

Bhabha, H. K. (2007). Les lieux de la culture : une théorie postcoloniale (traduit par F. Bouillot). Paris, France : Payot.

Buarque de Hollanda, Heloisa. (1997) O éthos Rachel. Dans Cadernos da Literatura Brasileira : Rachel de Queiroz (pp. 103-115). São Paulo, Brésil : Instituto Moreira Salles.

Condé, M. (2012). Moi, Tituba, sorcière... Noire de Salem. Paris, France : Gallimard.

Condé, M. (1986) [ $1^{\mathrm{e}}$ éd.]. Moi, Tituba, sorcière... Noire de Salem. Paris, France : Mercure de France.

Courteau, J. (2001). A feminização do discurso nacional na obra de Rachel de Queiroz. Hispania, 84 (4), 749-757. Disponible sur : http ://www.jstor.org/ $\underline{\text { stable } / 3657836}$

Foucault, M. (2001) [1984]. Des espaces autres. Dans Dits et écrits (pp. 15711581). Paris, France : Gallimard.

Fulton, D. (2008). Signs of Dissent : Maryse Condé and Postcolonial criticism. Charlottesville, VA : University of Virginia Press.

Jalaizal, Z. (2009). Historical Fiction and Maryse Condé's “I, Tituba, Black Witch of Salem". African American Review, 43 (2/3), 413-425. Disponible sur : http ://www.jstor.org/stable/41328618

Manzor-Coates, L. (1993). Of Witches and Other things : Maryse Condé's Challenges to Feminist Discourse. World Literature Today, 67 (4), 737 744. Disponible sur : http ://dx.doi.org/10.2307/40149572

Marouan, M. (2013). Witches, Goddesses, and Angry Spirits : The Politics of Spiritual Liberation in African Diaspora Women's Fiction. Columbus, OH : Ohio State University Press.

Martins Pereira, A. C. (2008) Recortes da obra Memorial de Maria Moura : o processo de (re)criação em cena. Maîtrise. Universidade Federal Fluminense.

Moss, J. (1999). Postmodernizing the Salem Witchcraze : Maryse Condé's I, Tituba, Black Witch of Salem. Colby Quarterly, 35 (1). Disponible sur : http ://digitalcommons.colby.edu/cq/vol35/iss1/3

Muniz de Albuquerque Jr., D. (1999). A invenção do nordeste e outras artes. São Paulo, Brésil : Cortez Editora.

Pfaff, F. (1993). Entretiens avec Maryse Condé. Paris, France : Karthala. 
Queiroz, R. de. (1992). Memorial de Maria Moura. São Paulo, Brésil : Editora Siciliano.

Queiroz, R. de. (1997). As três Rachéis. Entretien. Dans Cadernos da literatura brasileira. São Paulo, Brésil : Instituto Moreira Salles.

Queiroz, R. de (2009). Maria Moura (traduit par C. Tricoire) Paris, France : Métailié.

Rago, M. (1999). Introdução. Dans D. Muniz de Albuquerque Jr. A invenção do nordeste e outras artes. São Paulo, Brésil : Cortez Editora.

Sansavior, E. (2012). Voice, Irony and History in Moi, Tituba sorcière... Noire de Salem. Dans Maryse Condé and the Space of Literature (pp. 53-66). Wakefield, Royaume-Uni : MHRA et Maney Publishing.

Schpun, M. R. (2002). Lé com lé, cré com cré : fronteiras móveis e imutáveis em Memorial de Maria Moura. Dans L. Chiappini (éd.) et M. S. Bresciani (éd.). Literatura e cultura no Brasil : identidades e fronteiras (pp. 179-186). São Paulo, Brésil : Cortez Editora.

Smalls, J. (2004). Slavery Is a Woman : Race, Gender, and Visuality in Marie Benoist's Portrait d'une négresse (1800). Nineteenth-Century Art Worlwide, 3 (1). Disponible sur : http ://www.19thc-artworldwide.org/ spring04/70-spring04/spring04article/286-slavery-is-a-woman-racegender-and-visuality-in-marie-benoists-portrait-dune-negresse-1800

Tally, R. T. (2013). Spatiality. Londres, Royaume-Uni : Routledge.

Warf, B. et Arias, S. (2009). The Spatial Turn : Interdisciplinary Perspectives. Londres, Angleterre : Routledge.

West-Pavlov, R. (2009). Space in Theory : Kristeva, Foucault, Deleuze. Amsterdam, Pays-Bas : Editions Rodopi. 